\title{
Insulin resistance, serum insulin and HOMA-R
}

\author{
A. Campanati - G. Ganzetti · A. Di Sario • \\ A. Benedetti $\cdot$ A. Offidani
}

Received: 1 February 2013/Accepted: 5 February 2013/Published online: 12 March 2013

(C) Springer Japan 2013

\section{Dear Sir,}

The authors really appreciate the comments by Tomoyuki Kawada on their paper entitled "The effect of etanercept on hepatic fibrosis risk in patients with non-alcoholic fatty liver disease, metabolic syndrome and psoriasis" [1].

For Kawada [2], HOMA-R and serum insulin show equivalent efficacy in detecting insulin resistance, whereas it may be more difficult to calculate HOMA-R in subjects with increased fasting plasma glucose. Therefore, the homeostasis model assessment for insulin resistance could be substituted simply by serum insulin, with no need for data on plasma glucose [2].

This is a sharable point of view, however, the study reported by the author refers specifically to a wide male population without a current history of treatment for diabetes, hypertension, dyslipidemia, hyperuricemia, coronary, and/or cerebrovascular disease. Thus, when Kawada stated that both HOMA-R and serum insulin are equivalent in detecting insulin resistance, he specifically refers to apparently healthy subjects. However, conversely to population with no remarkable medical conditions considered by Kawada, our case series includes subjects with psoriasis,

This author's reply refers to the letter to the editor at doi:10.1007/s00535-013-0768-3.

A. Campanati · G. Ganzetti $(\bowtie) \cdot$ A. Offidani

Dermatologic Clinic,

Polytechnic University of Marche Region,

Ancona, Italy

e-mail: giulia.ganzetti@alice.it

A. Di Sario - A. Benedetti

Gastroenterologic Clinic,

Polytechnic University of Marche Region,

Ancona, Italy metabolic syndrome and NAFLD. In our opinion, in such a setting of patients, more than one marker of insulin resistance should be taken into consideration.

Regarding the second query, focusing on the use of QUICKI and HOMA indexes in our study, we have to specify that in our retrospective analysis we selected patients with concomitant psoriasis, metabolic syndrome and NAFLD who had received etanercept or PUVA, and did not base our selections exclusively on FPG values.

It's true that in our retrospective analysis, the FPG values of included patients ranged from 73 to $122 \mathrm{mg} / \mathrm{dl}$, but this was simply a matter of chance since we did not preventively exclude patients with FPG $>140 \mathrm{mg} / \mathrm{dl}$, as speculated by Kawada. Therefore, given FPG values $<140 \mathrm{mg} / \mathrm{dl}$, we deliberately decided to use both HOMA and QUICKI [3].

One of the limiting factors of our study was precisely that we did have not patients with FPG $>140 \mathrm{mg} / \mathrm{dl}$, because of its retrospective nature.

Finally, the authors acknowledge the suggestion to directly assess the risk of hepatic fibrosis in further advanced studies.

\section{References}

1. Campanati A, Ganzetti G, Sario AD, Damiani A, Sandroni L, Rosa L, Benedetti A, Offidani A. The effect of etanercept on hepatic fibrosis risk in patients with non-alcoholic fatty liver disease, metabolic syndrome, and psoriasis. J Gastroenterol. 2012 Oct 13. doi:10.1007/s00535-012-0678-9.

2. Kawada T. Preliminary report: homeostasis model assessment of insulin resistance, an indicator of insulin resistance, is strongly related to serum insulin: practical data presentation and the mathematical basis. Metabolism. 2010;59(7):1044-6.

3. Martinez-Hervas S, Argente C, Garcia-Jodar J, Priego A, Real JT, Carratala A, et al. Misclassification of subjects with insulin resistance and associated cardiovascular risk factors by homeostasis model assessment index. Utility of a postprandial method based on oral glucose tolerance test. Metabolism. 2011;60(5):740-6. 